\title{
Forward energy measurement with CMS
}

\author{
Lev Kheyn ${ }^{\mathrm{a}}$ for the CMS Collaboration \\ Skobeltsyn Institute of Nuclear Physics, Lomonosov Moscow State University, Russia
}

\begin{abstract}
Energy flow is measured in the forward region of CMS at pseudorapidities up to 6.6 in pp interactions at $13 \mathrm{TeV}$ with forward (HF) and very forward (CASTOR) calorimeters. The results are compared to model predictions. The CMS results at different centre-of-mass energies are compared using the pseudorapidity variable shifted by the beam rapidity, thus studying applicability of the hypothesis of limiting fragmentation.
\end{abstract}

\section{Introduction}

An almost hermetic calorimetric instrumentation of the CMS experiment extends from $\eta=-6.6$ to 5.2 (for neutral particles also at $|\eta|>8.1$ ). This makes it possible to perform energy measurements in a wide range of pseudorapidities. Such measurements allow to explore basic characteristics of the underlying event and multiple parton interactions (MPI) as a function of pseudorapidity and center-of-mass energy. For models used in cosmic ray physics, data at collider energies are an important reference necessary for extrapolating to highest energies.

At the LHC, energy flow measurements have been performed by ATLAS, LHCb, and CMS collaborations. ATLAS and $\mathrm{LHCb}$ performed measurements in $\mathrm{pp}$ collisions at $\sqrt{s}=7 \mathrm{TeV}$ in the pseudorapidity ranges $|\eta|<4.8$ [1] and $1.9<|\eta|<4.9$ [2], respectively. CMS performed studies in pp collisions at 0.9 and $7 \mathrm{TeV}$ [3], as well as in collisions of lead ions at $2.76 \mathrm{TeV}$ [4] and proton-lead-ion collisions at 5.02 $\mathrm{TeV}$ [5].

In this work, we present results of measurements of the dependence of the energy flow on pseudorapidity at $3.15<|\eta|<6.6$ performed with use of the HF and CASTOR calorimeters [6], and the energy distribution in the CASTOR calorimeter [7].

\section{CMS detector}

A central part of the Compact Muon Solenoid (CMS) apparatus is a superconducting solenoid of $6 \mathrm{~m}$ internal diameter, providing a strong magnetic field of $3.8 \mathrm{~T}$. Within the field volume are an inner silicon pixel and strip tracker that measures charged particles in the range $|\eta|<2.5$, a homogeneous lead tungstate crystal electromagnetic calorimeter, and a brass and scintillator hadron calorimeter. Muons are measured in gas-ionization detectors embedded in the steel return yoke. Extensive forward calorimetry complements the coverage provided by the barrel and endcap detectors. For more details on CMS see [8].

Cherenkov calorimetry was chosen in CMS for the forward detectors. The HF calorimeters cover $2.9<$ $|\eta|<5.2$ and use quartz fibers embedded within a steel

a e-mail: kheyn@mail.cern.ch absorber. The HF is segmented in 13 towers in $\eta$, each with a size given by $\Delta \eta \simeq 0.175$, except for the lowest and highest- $|\eta|$ towers with $\Delta \eta \simeq 0.1$ and $\Delta \eta \simeq 0.3$, respectively. The azimuthal segmentation of all towers is $10^{\circ}$, except for the one at highest- $|\eta|$, which has $\Delta \varphi=20^{\circ}$. The very forward angles are covered by the CASTOR $(-6.6<\eta<-5.2)$ calorimeter which is made of quartz plates embedded in tungsten absorbers. The CASTOR has 16 azimuthal sectors $\left(22.5^{\circ}\right.$ each $)$ and 14 longitudinal modules ( 2 modules in the electromagnetic section, 10 radiation lengths each, and 12 modules in the hadronic section, twice the depth of electromagnetic modules each). The overall longitudinal depth of both CASTOR and HF corresponds to 10 nuclear interaction lengths.

\section{Event selection}

Two different strategies are used for selection of inelastic collision events: an inclusive selection, allowing significant fraction of diffractive events, and a non-singlediffractive selection where single-diffractive contribution is effectively suppressed. This is achieved by requiring activity in HF either on at least one side (for the former) or on both sides (for the latter) with respect to the nominal interaction point of CMS.

In the analysis, activity in HF is identified by presence of a reconstructed calorimeter tower with energy well above noise level. For selection of the inclusive inelastic events, the cut $5 \mathrm{GeV}$ is used. For the non-single-diffractive selection, due to reduced sensitivity to noise, a lower cut of $4 \mathrm{GeV}$ is chosen.

The data are corrected to the hadron level using Pythia8 Monash, Pythia8 MBR, EPOS-LHC and QGSJET-II-04 MC generators together with a simulation of the CMS detector based on GEANT4. The hadron level energy is a sum of the energies of stable particles, with exclusion of muons and neutrinos, within the pseudorapidity range of the measurement $(3.15<\eta<6.6)$ without any threshold. Corrections are applied on a bin-by-bin basis. The correction factor is calculated as a ratio of the average MC predictions at the hadron level to the average predictions at the detector level. Final correction factor is the average of the correction factors for four different MC samples. 

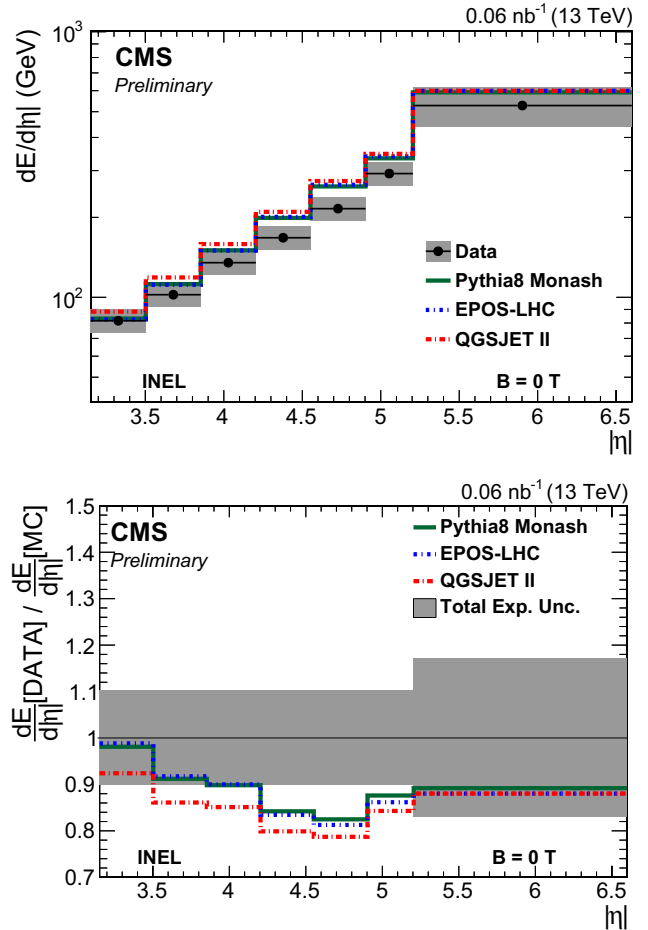

Figure 1. A comparison of the energy flow measurements as a function of pseudorapidity at $13 \mathrm{TeV}$ to MC models [6]. The gray band shows the total systematic uncertainty correlated across pseudorapidity bins. The lower panel shows the ratio of measured data to MC predictions.

Selection of events on the hadron level is done differently for soft-inclusive-inelastic sample and for nonsingle-diffractive sample. Soft-inclusive-inelastic events are selected with use of the cut $\xi=\max \left(\xi_{X}, \xi_{Y}\right)>10^{-6}$. Here $\xi_{X}=M_{X}^{2} / s$ and $\xi_{Y}=M_{Y}^{2} / s$ where $M_{X}$ and $M_{Y}$ are invariant masses of two systems of stable particles that are separated by the largest rapidity gap in the event. Nonsingle-diffractive events are selected with a requirement of at least one particle except muon or neutrino within $\eta$ acceptance of the HF calorimeters at both sides. Also, for comparison to previously published results [3], nonsingle-diffractive events are selected with a requirement of at least one charged particle at both sides of the CMS in the pseudorapidity range $3.9<|\eta|<4.4$.

A dominant systematic effect which limits accuracy of the measurement of the forward energy flow is the global energy scale uncertainty of the forward calorimeters, which is estimated to be $\pm 10 \%$ and $\pm 17 \%$ for $\mathrm{HF}$ and CASTOR calorimeters, respectively.

\section{Results and discussion}

The energy and transverse energy densities, $d E / d \eta$ and $d E_{T} / d \eta$, measured using the CMS HF and CASTOR forward calorimeters and corrected to the particle level, are presented in the pseudorapidity range $3.15<|\eta|<6.6$.

A comparison of the measured energy flow to $\mathrm{MC}$ model predictions for the soft-inclusive inelastic selection is shown in Figs. 1-2. The gray band represents the total systematic uncertainty correlated across pseudorapidity bins. The statistical uncertainties are negligible and not shown. The red band indicates the envelope of the uncertainties of the parameters of Pythia8 CUETP8S1
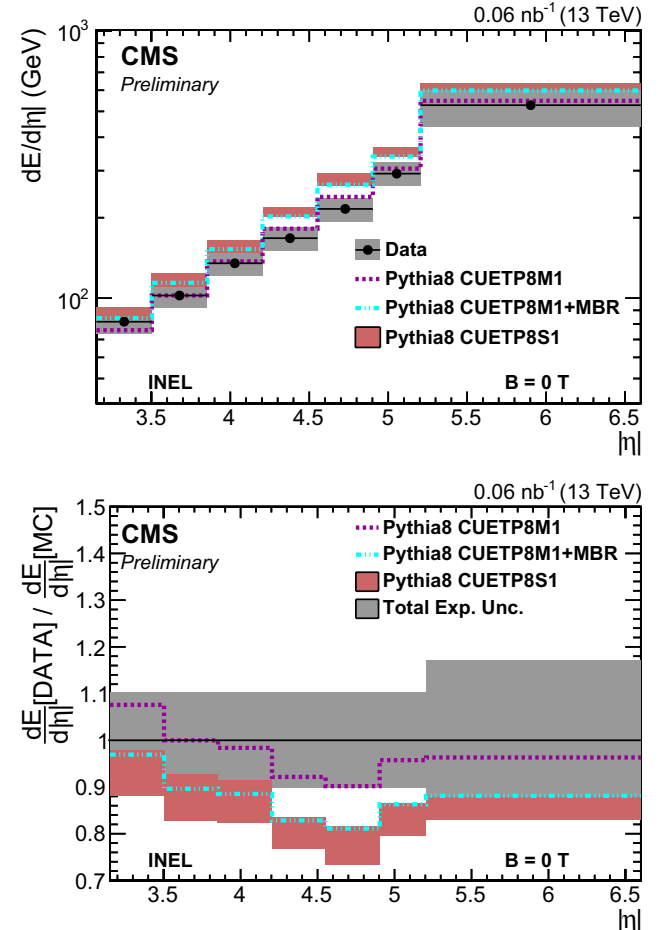

Figure 2. A comparison of the energy flow measurements as a function of pseudorapidity at $13 \mathrm{TeV}$ for soft-inclusiveinelastic events to MC models at particle level [6]. The gray band shows the total systematic uncertainty correlated across pseudorapidity bins. The red band corresponds to the envelope of the uncertainties of the parameters of Pythia8 CUETP8S1 tune. The lower panel shows the ratio of measured data to MC predictions.

tune. In the upper panels the comparison is shown in absolute values, while in the lower panels the ratios quantify the agreement of the measurements and MC predictions.

There is a large spread of the model predictions in the region of the HF coverage, $3.15<|\eta|<5.20$. At low $|\eta|$ predictions are consistent with the data, most of the models overestimate the measurements towards higher $|\eta|$ values, and become again closer to the data in the CASTOR bin, $5.2<|\eta|<6.6$. The best description of the data is provided by the Pythia8 tune CUETP8M1.

In Figs. 3-4, energy flow measurements are confronted to the MC model predictions for the non-single-diffractiveenhanced event selection. In the upper panels the comparison is shown in absolute values, while in the lower panels the ratios quantify the agreement of the measurements and MC predictions. For non-single-diffractive-enhanced events, the spread of the model predictions is smaller than that for soft-inclusive-inelastic events. The EPOS-LHC and QGSJETII-04 MC generators provide good overall description of the data in the whole $|\eta|$ range of the measurements. The Pythia8 tune CUETP8M1 provides satisfactory description of the data, significantly deviating from the measurements only at low $|\eta|$.

In the lower panel of Figs. 3-4, the ratio is also shown of the energy flow for non-single-diffractive- enhanced to that for soft-inclusive-inelastic events. No significant difference in the slope of the pseudorapidity dependence is observed. 

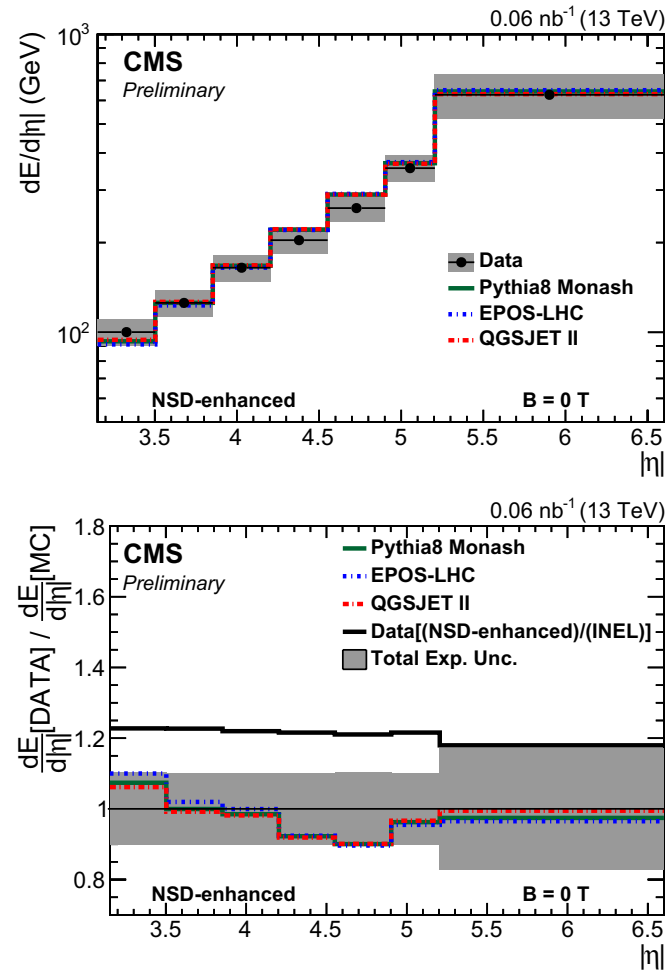

Figure 3. A comparison of the energy flow measurements as a function of pseudorapidity at $13 \mathrm{TeV}$ for non-single-diffractiveenhanced events to MC models at particle level [6]. The gray band shows the total systematic uncertainty correlated across pseudorapidity bins. The lower panel shows the ratio of measured data to MC predictions and, as a solid black line, ratio of the results for non-single-diffractive-enhanced (NSD-enhanced) and soft-inclusive-inelastic (INEL) events.

Figure 5 presents a comparison of the transverse energy density, $\mathrm{d} E_{T} \mathrm{~d} \eta^{\prime}$, with published CMS data at lower $\sqrt{s}$ and results at $13 \mathrm{TeV}$ with models as a function of shifted pseudorapidity variable $\eta^{\prime}=\eta-y_{\text {beam }}$. Nonsingle-diffractive events are selected with requirement of at least one charged particles in the range $3.9<|\eta|<4.4$ on each side of CMS. Here $y_{\text {beam }}$ stands for the beam rapidity.

The models provide overall reasonable description of the data. The results at different energies are shown to be consistent in terms of shifted pseudorapidity variable, $\eta-y_{\text {beam }}$, and consistent with the hypothesis of limiting fragmentation.

In Figure 6, the distribution of the total energy in the CASTOR calorimeter is shown for soft-inclusive-inelastic events. Despite significant systematic uncertainties, mainly due to the energy scale, model predictions show clear differences with the measured data. The bump at a few hundred $\mathrm{GeV}$ is also visible in all model predictions. It is best described by QGSJETII-04. The Pythia8 tunes tend to overestimate the contribution of this soft part to the spectrum. The high energy tail on the other hand is described by all models within the experimental uncertainties. Only QGSJETII-04 has a too flat total and hadronic spectrum in the range $0.5 \mathrm{TeV}<\mathrm{E}<1.8 \mathrm{TeV}$ and a too steeply falling spectrum above $1.8 \mathrm{TeV}$. The data is also very sensitive to MPI and the underlying event.

\section{Summary}

A measurement of the energy flow is presented in the pseudorapidity range $3.15<|\eta|<6.6$ in proton-proton
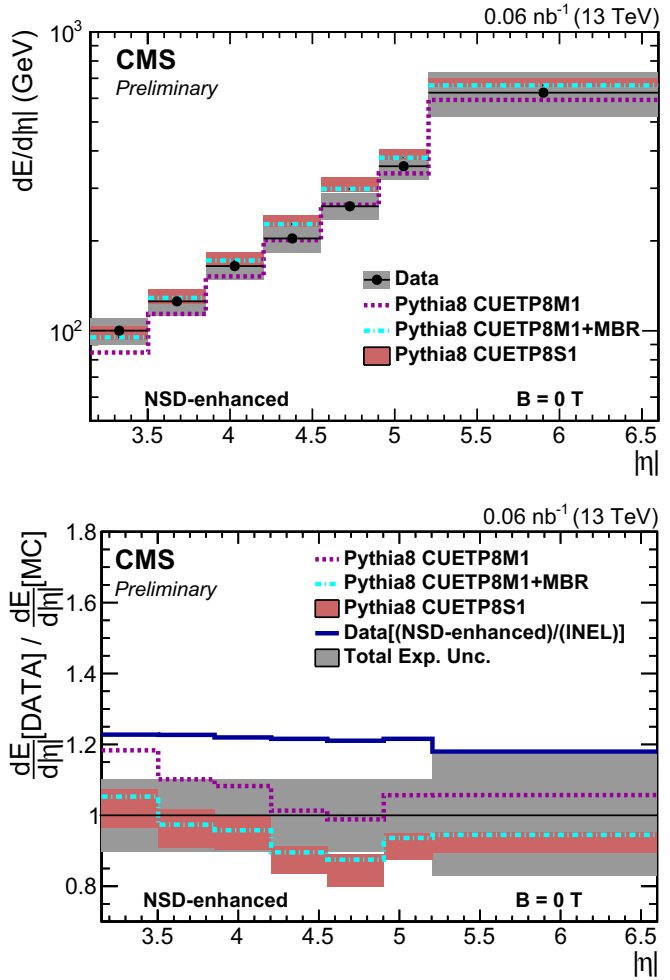

Figure 4. A comparison of the energy flow measurements as a function of pseudorapidity at $13 \mathrm{TeV}$ for non-single-diffractiveenhanced events to MC models at particle level [6]. The gray band shows the total systematic uncertainty correlated across pseudorapidity bins. The red band corresponds to the envelope of the uncertainties of the parameters of Pythia8 CUETP8S1 tune. The lower panel shows the ratio of measured data to MC predictions and, as a solid black line, ratio of the results for nonsingle-diffractive-enhanced (NSD-enhanced) and soft-inclusiveinelastic (INEL) events.

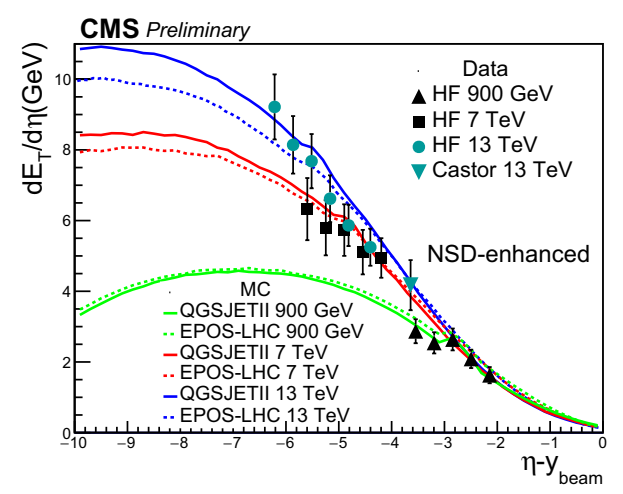

Figure 5. A comparison of the measured hadron level transverse energy density $\mathrm{d} E_{T} \mathrm{~d} \eta^{\prime}$ to the published CMS measurements and several models at different $\sqrt{s}$ as function of the shifted pseudorapidity variable, $\eta-y_{\text {beam }}$ [6]. The hadron level definition is according to the CMS published results [3].

collisions for the centre-of-mass energy of $\sqrt{(} s)=$ $13 \mathrm{TeV}$. The energy flow is studied as a function of pseudorapidity for soft-inclusive-inelastic and non-singlediffractive-enhanced events. The results are compared to several Pythia8 tunes, EPOS-LHC and QGSJETII-04 hadronic interaction models, commonly used in cosmic ray physics, and earlier pp data at $\sqrt{s}=900 \mathrm{GeV}$ and $7 \mathrm{TeV}$. The considered models provide in general reasonable 

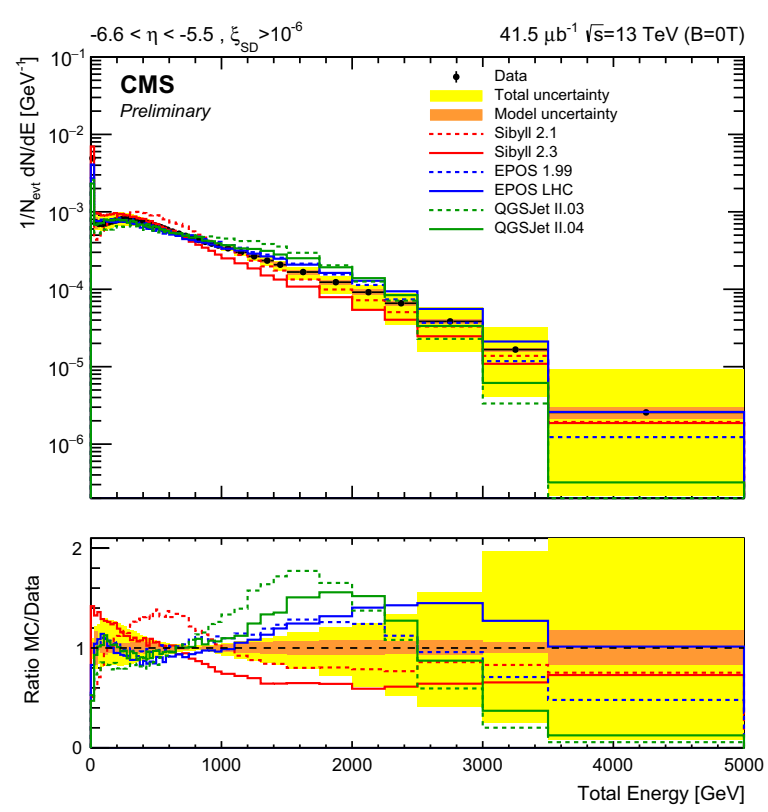

Figure 6. Normalized total energy spectrum in the CASTOR for events with $\xi>10^{-6}[7]$.

description of the measured energy flow at the highest energy achieved so far at the LHC. The spread of the model predictions for soft-inclusive-inelastic events is large and becomes smaller for non-single-diffractiveenhanced events. The results at different energies are shown to be consistent in terms of shifted pseudorapidity variable $\eta^{\prime}=\eta-y_{\text {beam }}$, and reveal overall consistency with the hypothesis of limiting fragmentation in a wide range of pp collision energies from $900 \mathrm{GeV}$ to $13 \mathrm{TeV}$.

Model predictions show clear differences from the measurements in the distribution of the total CASTOR calorimeter energy.

\section{References}

[1] ATLAS Collaboration, JHEP 11 (2012) 033, arXiv:1208.6256

[2] LHCb Collaboration, Eur. Phys. J. C 73 (2013) 2421, arXiv: 1212.4755

[3] CMS Collaboration, JHEP 11 (2011) 148, JHEP02 (2012) 055, arXiv:1110.0211. [Erratum: JHEP02, 055 (2012)]

[4] CMS Collaboration, CMS Physics Analysis Summary CMS-PAS-HIN-12-006, 2012

[5] CMS Collaboration, CMS Physics Analysis Summary CMS-PAS-HIN-14-014, 2015

[6] CMS Collaboration, CMS Physics Analysis Summary CMS-PAS-FSQ-15-006, 2015

[7] CMS Collaboration, CMS Physics Analysis Summary CMS-PAS-FSQ-16-002, 2016

[8] CMS Collaboration, JINST 3 (2008) S08004 